\title{
Gaia pulsars and where to find them
}

\author{
John Antoniadis ${ }^{1,2,3 \star}$ \\ ${ }^{1}$ Max-Planck-Instutut für Radioastronomie, Auf dem Hügel 69, 53121, Bonn, DE \\ ${ }^{2}$ Argelander Institut für Astronomie, Auf dem Hügel 71, 53121, Bonn, DE \\ ${ }^{3}$ Institute of Astrophysics, FORTH, Dept. of Physics, University Campus, GR-71003 Heraklion, Greece
}

17 November 2020

\begin{abstract}
While the majority of massive stars have a stellar companion, most pulsars appear to be isolated. Taken at face value, this suggests that most massive binaries break apart due to strong natal kicks received in supernova explosions. However, the observed binary fraction can still be subject to strong selection effects, as monitoring of newly-discovered pulsars is rarely carried out for long enough to conclusively rule out multiplicity. Here, we use the second Gaia Data Release (DR2) to search for companions to 1534 rotation-powered pulsars with positions known to better than 0 .' 5 . We find 22 matches to known pulsars, including 1 not reported elsewhere, and 8 new possible companions to young pulsars. We examine the photometric and kinematic properties of these systems and provide empirical relations for identifying Gaia sources with potential millisecond pulsar companions. Our results confirm that the observed multiplicity fraction is small. However, we show that the number of binaries below the sensitivity of Gaia and radio timing in our sample could still be significantly higher. We constrain the binary fraction of young pulsars to be $f_{\text {young }}^{\text {true }} \leq 5.3(8.3) \%$ under realistic(conservative) assumptions for the binary properties and current sensitivity thresholds. For massive stars $\left(\geq 10 \mathrm{M}_{\odot}\right)$ in particular, we find $f_{\mathrm{OB}}^{\text {true }} \leq 3.7 \%$ which sets a firm independent upper limit on the galactic neutron-star merger rate, $\leq 7.2 \times 10^{-4} \mathrm{yr}^{-1}$. Ongoing and future projects such as the CHIME/pulsar program, MeerTime, HIRAX and ultimately the SKA, will significantly improve these constraints in the future.
\end{abstract}

Key words: stars: neutron — stars: pulsars - stars: massive - miscellaneous — catalogs — surveys

\section{INTRODUCTION}

Multiplicity plays a critical role in the evolution of massive stars: interactions with a stellar companion can modify a star's mass, composition and angular momentum, thereby influencing the manner in which it dies, and the type of remnant it produces. Studies of star-forming associations suggest that most young massive stars $(\sim 100 \%)$ have a gravitationally-bound companion, with the majority $(\sim 70 \%)$ being close enough to eventually interact via mass exchange (Sana et al. 2012). Such interactions can lead to stellar mergers, X-ray binaries (XRBs), supernovae ( $\mathrm{SNe}$ ) and hyper-velocity stars. Systems that survive these processes and remain bound ultimately become compact-object binaries and gravitational wave (GW) sources.

Probing how the multiplicity fraction evolves as a stellar population ages is crucial for understanding these astrophysical phenomena and the underlying physical processes, including natal SN kicks (Bailes 1989; Portegies Zwart \& Yungelson 1999; Janka 2013), the physics of common envelope (CE) evolution (Ivanova et al. 2013), and the birth vs remnant mass relation (Antoniadis et al. 2016; Tauris et al. 2017).

^ E-mail: janton@mpifr.de
Theoretical models and predictions should confront to a number of observational benchmarks, such as the distribution of pulsar velocities (Hobbs et al. 2005), the number of XRBs and their relative distances from star-forming regions (Walter et al. 2015; Tauris et al. 2017), the birth rates and properties of double neutron star systems (Kalogera et al. 2004; Tauris et al. 2017; Ferdman et al. 2020), and the observed GW merger rates (Abadie et al. 2010; Abbott et al. 2017). One important independent constraint that remains underutilized is the multiplicity fraction among young, non-recycled pulsars (Bailes 1989). The frequency of binary systems that survive SN explosions is a sensitive probe of the SN kick magnitude distribution, which remains one of the major theoretical uncertainties. There are only a handful of known binaries hosting young radio pulsars, implying that most $\mathrm{SNe}$ result in disruptions. However, the observed multiplicity fraction is likely subject to significant selection effects as most new pulsar discoveries are typically monitored only for up to a few months. Owing to the presence of timing noise and the strong co-variances with the intrinsic spin-down, such observations are not particularly sensitive to orbital acceleration caused by lowmass companions and/or wide binary pairs. Indeed, even some of the few known binaries have been discovered only by chance (Lyne et al. 2015; Kaplan et al. 2016; Bassa et al. 2016).

In this work we revisit the constraints on pulsar multiplicity 


\section{J. Antoniadis}

using the second Gaia data release (DR2; Gaia Collaboration et al. 2016; Lindegren et al. 2018) which, for the first time, provides information on the distances and kinematic properties of nearly two billion stars in the Galaxy and Magellanic Clouds. In Section 2 we describe our sample and methodology, while in Section 3 we present our results. In Section 4 we examine the influence of various selection effects and current limitations, and derive a constraint on the multiplicity fraction of young pulsars. We conclude with a discussion in Section 5.

\section{METHODS}

\subsection{Sample}

In what follows, we distinguish between different classes of rotationpowered pulsars based on their inferred minimum dipole magnetic field strength, $(B / G) \equiv 3.2 \times 10^{19}\left(P \dot{P} s^{-1}\right)^{1 / 2}$. More specifically, sources with $(B / \mathrm{G}) \leq 10^{9}$ are considered fully-recycled millisecond pulsars (MSPs), the ones with $10^{9}<(B / \mathrm{G}) \leq 10^{10}$ are mildly-recycled pulsars (henceforth mild MSPs), while those with $(B / G)>10^{10}$ are referred to as young, non-recycled pulsars.

Our main goal is to identify possible companions to known rotation-powered pulsars. There are currently 2492 pulsars outside of globular clusters listed in the ATNF pulsar catalogue ${ }^{1}$ (PSRCat v1.63; Manchester et al. 2005). Since we are primarily interested in finding close astrometric pairs, we only considered those with positions known to better than 0 .' 5 . This leaves 1534 objects (see Figure 1), a sample considerably larger than those used in previous searches for Gaia counterparts (65 MSPs in Mingarelli et al. 2018, 155 binaries in Jennings et al. 2018 and 57 non-recycled pulsars in Igoshev \& Perets 2019). Of these, 107 are MSPs, 39 are mild MSPs and 1388 are young pulsars. The sample contains 145 known binaries (86 MSPs, 32 mild MSPs and 27 young pulsars) and 11 extra-galactic sources.

\subsection{Identification of Candidate Astrometric Pairs}

We searched for possible pulsar companions among Gaia DR2 objects (Lindegren et al. 2018) located within 20" of each pulsar. This search radius is equivalent to the angular distance that would be covered by an object at $500 \mathrm{pc}$, moving with a transverse velocity of $v_{\text {tr }}=1200 \mathrm{~km} \mathrm{~s}^{-1}$ for $40 \mathrm{yr}$. For each Gaia entry, we used the available astrometry to propagate the celestial coordinates and their uncertainties to the reference epoch of the pulsar ephemeris (PosEpoch in PSRCat). This choice allowed us to utilize the entire sample instead of only the subset of pulsars with timing proper motions. For the few sources for which only a two-parameter astrometric solution is available, we used directly their Gaia epoch position (J2015.5). Sources outside the $2 \sigma$ positional error circle of the pulsar were dismissed as unrelated. For each possible astrometric pair, we inferred a probability of chance coincidence using the average number density of stars within $1^{\prime}$ of the pulsar (Antoniadis et al. 2011).

Given that many pulsar and Gaia DR2 positions considered here are known to $\ll 0$.' 5 precision, their uncertainties are likely dominated by systematics. One important source of potential errors is rotations between the Gaia reference frame - which is tied to the International Celestial Reference Frame (ICRS, see Mignard

${ }^{1}$ http://www.atnf.csiro.au/research/pulsar/psrcat accessed on Oct 202020

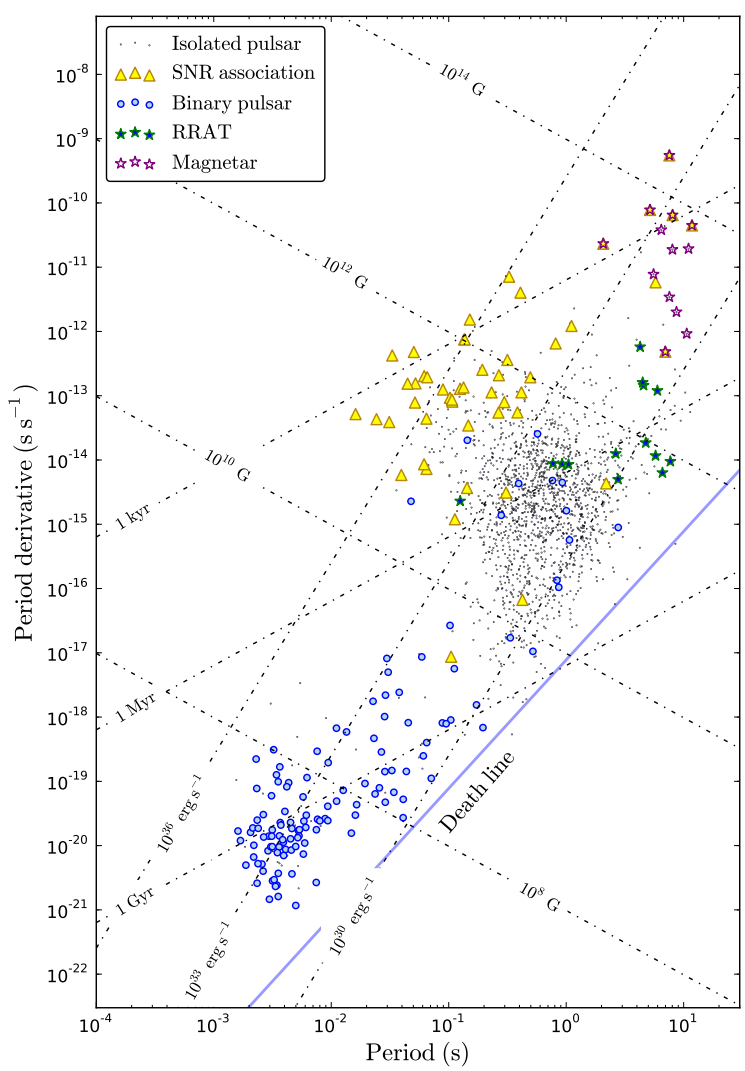

Figure 1. $\mathrm{P}-\dot{P}$ diagram of the pulsars contained in our sample.

et al. 2018) - and the various incarnations of the Solar System ephemeris utilized in pulsar timing. Co-variances between astrometric and other parameters in the pulsar timing solution, red noise in the Gaia astrometry and timing noise may play a role as well. Here, we did not attempt to correct explicitly for these effects. Instead, we compensated for them by adding 0. " 25 in quadrature to the inferred position error. This value was adopted solely based on empirical considerations. More specifically, it corresponds to $\sim 1 / 2$ of the second largest separation between a pulsar and its known optical counterpart (PSR J0045-7319; see Table 1 and Section 3.2). This choice also made our search sensitive to nearby astrometrically-resolved binaries that would have been missed otherwise ( $0 .{ }^{\prime \prime} 25$ is equivalent to a separation of $125 \mathrm{AU}$ at $500 \mathrm{pc}$, see Section 3.1). Increasing the systematic uncertainty beyond this value - for instance, to include PSR J2129-0429 for which the inferred separation is $\sim 1$." 4 - resulted in a very large number of false positives.

\subsection{Distances, Reddening and Kinematics}

It is well known that obtaining distance estimates by directly inverting the parallax measurement can lead to significant bias in the presence of noise. Following previous work (Jennings et al. 2018) and recommendations (Bailer-Jones 2015; Astraatmadja \& BailerJones 2016; Bailer-Jones et al. 2018; Luri et al. 2018), we inferred 
probability density functions (PDFs) for the distance to sources of interest using:

$$
P(d \mid \hat{\pi}) \propto \frac{1}{2 L^{3}} e^{-d / L} d^{2} e^{(-1 / d-\hat{\pi})^{2} / 2 \sigma_{\hat{\pi}}^{2}},
$$

Here, we assume that the parallax measurement, $\hat{\pi}$ is normally distributed about the true parallax, $1 / d$, with a dispersion $\sigma_{\hat{\pi}}$. The $e^{-d / L} d^{2} L^{-3}$ term accounts for a Lutz-Kerker bias (Lutz \& Kelker 1973) with an exponentially-decreasing stellar density (that is nearly constant for $d \ll L$ ). The former prior combined with the condition $d>0$ has the advantage of making the distance PDF well-behaved and normalisable so that statistical moments can be defined. For our purposes, distances are not the primary quantity of interest and thus $L$ was simply taken to be direction-independent and was set equal to $1.5 \mathrm{kpc}$ (Bailer-Jones et al. 2018). This should be adequate for the majority of sources with small Galactic latitudes, but may overestimate the distances to sources with imprecise parallaxes located outside the Galactic plane. For more detailed models, see Jennings et al. (2018); Bailer-Jones (2015) and references therein.

When applicable, we also made use of other available measurements that contain distance information. More specifically, for certain pulsar/Gaia cross-matches we utilized timing and VLBI parallaxes (inverted using Eq. 1), and DM-based distance estimates derived using the NE2001 model and assuming a $20 \%$ uncertainty (Deller et al. 2019; Ding et al. 2020). For extra-galactic sources we used the most recent distance estimates to the Large(Small) Magellanic Cloud, 49.97 $\pm 1.13(62.1 \pm 1.9)$ kpc (Pietrzyński et al. 2013; Graczyk et al. 2013). For non-certain associations, we simply required the distance be positive. In what follows we report median values as point estimators together with $16 \%$ and $68 \%$ percentiles as uncertainties.

The interstellar reddening along each line of sight was traced using the latest version of the 3D dust map of Green et al. (2019) (Bayestar $19^{2}$; see also Green et al. 2014; Green 2018). Finally we derived estimates for the reduced proper motion (Luyten 1922) using,

$$
H_{\mathrm{g}}=m_{\mathrm{g}}+5 \log _{10} \frac{\mu}{\text { mas yr }^{-1}}-10+A_{\mathrm{g}}
$$

where $m_{\mathrm{g}}$ and $A_{\mathrm{g}}(d)$ are the apparent magnitude and extinction in the Gaia band, and $\mu=\sqrt{\mu_{\alpha}^{2}+\mu_{\delta}^{2}}$ is the magnitude of the proper motion. This quantity is analogous to the absolute magnitude but sensitive to the source's transverse velocity, $H_{\mathrm{g}} \equiv$ $M_{\mathrm{g}}+5 \log _{10}\left(v_{\mathrm{tr}} / 4.74057 \mathrm{~km} \mathrm{~s}^{-1}\right)$. This makes it a useful quantity for distinguishing between different kinematic populations, even in the absence of a precise parallax measurement.

\section{RESULTS}

Our search identified 32 close astrometric pairs with a probability of chance coincidence smaller than $10 \%$. These sources include 22 known binaries, 8 new candidate counterparts, the Crab pulsar (PSR B0531+21) and the pulsar wind nebula of PSR B0540-69 in the LMC. For the majority of these sources, the inferred probability of chance coincidence is $1-3 \%$, implying that the number of false positives in this sample is $O(3)$ or smaller.

The properties these Gaia sources are listed in Table 1, while Figure 2 shows their location on color-magnitude (henceforth HR) and color- $\mathrm{H}_{\mathrm{g}}$ (henceforth RPM) diagrams.
As can be seen, for most confirmed pulsars, the angular separation $\hat{\theta}$ at PosEpoch is much smaller than the position error, which is dominated by the systematics. Two notable exceptions are PSR J0045-7319, for which the timing solution has not been updated for over 25 years (Kaspi et al. 1996a), and PSR J0437-4715, a nearby MSP. The reason for the large discrepancy in the latter case is not clear, but it might be related to the faintness of the optical counterpart and the proximity of the system to Earth.

In the rest of this section we discuss the properties of confirmed matches and candidates in more detail.

\subsection{Millisecond Pulsars}

We found 18 matches to known MSPs including PSR J0348+0432 which, even though does not satisfy our formal MSP definition, is most likely a fully-recycled pulsar (Antoniadis et al. 2013). With the exception of PSRs B1953+29, B1957+20 and J1435-6100, these Gaia counterparts have also been identified by Jennings et al. (2018), Mingarelli et al. (2018) and Igoshev \& Perets (2019).

The majority of these sources are eclipsing MSPs with stellaror Jupiter- mass companions (often referred to as "redbacks" and "black widows" respectively). As can be seen in Figure 2 these sources occupy sparsely populated regions on the HR and RPM diagrams: they are both bluer and significantly brighter compared to MS stars of comparable masses. This supports the idea that they either have a high helium content (i.e. they are stripped), or that their atmospheres are highly irradiated. On the RPM diagram, MSP companions are even more separated from MS stars. On average, they have large proper-motion moduli, suggestive of high velocities that are likely a fossil signature of the SN explosion. We find that most eclipsing MSPs, as well as three MSPs with low-mass white dwarf (WD) companions, satisfy the following conditions:

$$
\begin{aligned}
M_{\mathrm{G}} & \leq 2.7\left(G_{\mathrm{BP}}-G_{\mathrm{RP}}\right)+10.5, \\
M_{\mathrm{G}} & >3.7\left(G_{\mathrm{BP}}-G_{\mathrm{RP}}\right)+2.4, \\
H_{\mathrm{g}} & >5.3\left(G_{\mathrm{BP}}-G_{\mathrm{RP}}\right)+5.9,
\end{aligned}
$$

where $G_{\mathrm{BP}}$ and $G_{\mathrm{RP}}$ are the mean magnitudes in the Gaia BP and RP photometric bands. These empirical criteria are efficient in filtering out $99.8 \%$ of the field stars shown in Figure 2. They are also robust against selecting high-velocity MS stars with similar $H_{\mathrm{g}}$ values. However, some overlap is expected with early-type subdwarfs and extremely-low mass white dwarfs and their precursors (Pelisoli \& Vos 2019; Pelisoli et al. 2019a). Utilizing color and $\mathrm{H}_{\mathrm{g}}$ information only (i.e. the last two relations) results in significant overlap with WDs and higher contamination from halo stars (at the $\sim 1-2 \%$ level), but has the benefit of being applicable to sources for which a reliable parallax measurement is not available. This can be compensated by additional criteria (e.g. $H_{\mathrm{g}} \leq 6.9\left(G_{\mathrm{BP}}-G_{\mathrm{RP}}\right)+$ 13.0) to filter out WDs, or multi-wavelength information (e.g. the presence of X-ray, $\gamma$-ray or radio counterparts). We provide some practical examples for identifying pulsar candidates in a python notebook accompanying this paper ${ }^{3}$.

\section{Individual sources and comparison to previous work}

PSR J1024-0719 This MSP was long thought to be isolated. However Bassa et al. (2016) and Kaplan et al. (2016) identified $\mathrm{a} \sim 0.4 \mathrm{M}_{\odot}$ MS K-dwarf as a potential companion in a very long period (2-20 kyr) orbit. Our search identified 

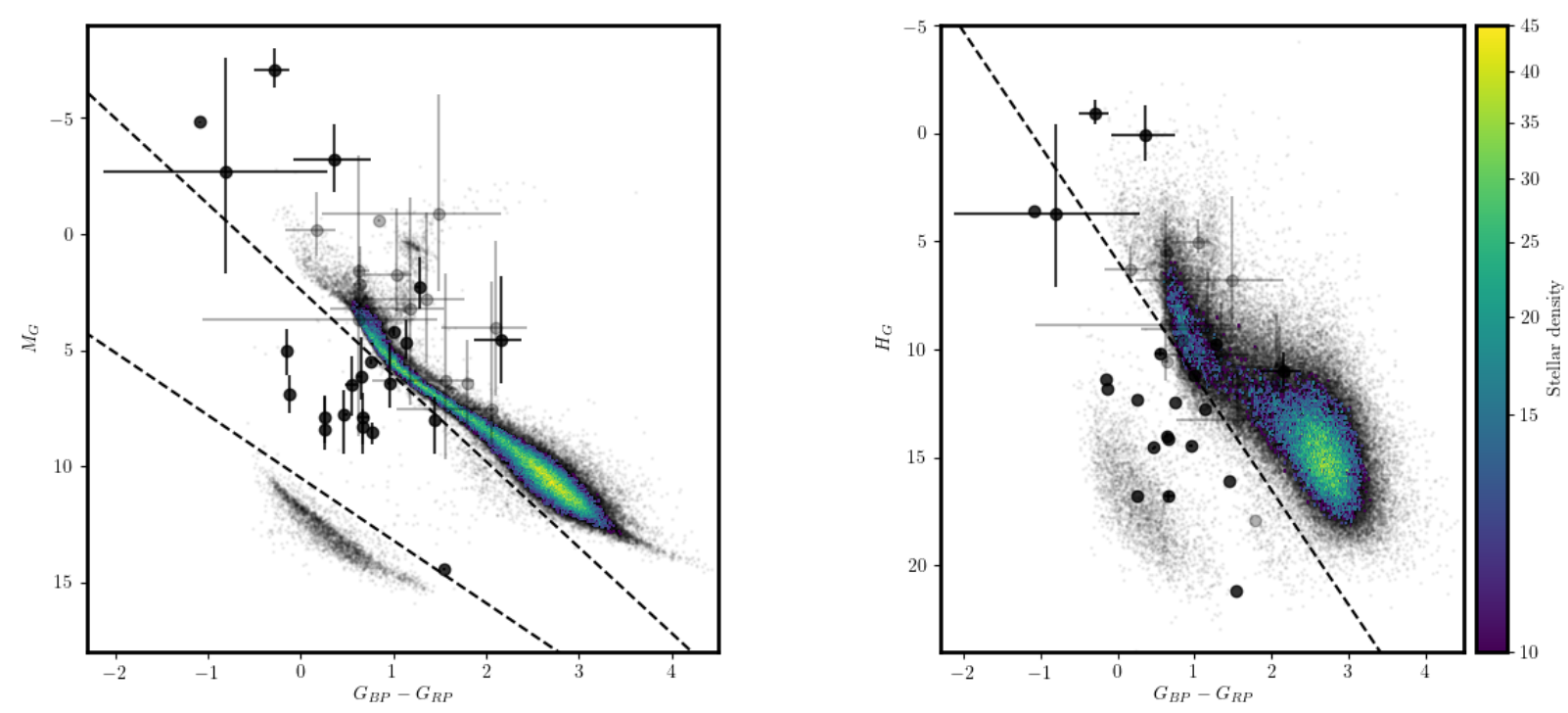

Figure 2. Positions of known and candidate pulsar companions and with Gaia counterparts on the observational HR (left) and RPM diagrams (right). Scatter points and the overlaid histogram shows field stars with precise parallax measurements within 200 pc from the Sun. The positions of known pulsars and candidate associations are shown in black and gray respectively. The dashed lines show the empirical cuts of Eq. 3 (see text for details).

Gaia 3775277872387310208 as the pulsar companion (see also Jennings et al. 2018) with a true angular separation of $\theta=0 !^{\prime \prime} 076(3)$ (without accounting for systematic uncertainties which are likely not dominant in this case). At the inferred distance of $3.4_{-2.0}^{+4.6} \mathrm{kpc}$, this implies a projected separation of $260_{-195}^{+852} \mathrm{AU}$, or an orbital period between $\sim 0.75$ and $12 \mathrm{kyr}$. The uncertainty is clearly dominated by the distance estimate and thus it will improve significantly with further timing observations and the next Gaia data release. This source clearly demonstrates the complementary of optical astrometry and radio timing for identifying binary pulsars.

PSR B1957+20 This pulsar is the prototypical "black widow" system, a 2-ms MSP with a Jupiter-mass companion in a $9 \mathrm{~h}$ orbit. Jennings et al. (2018) discuss a counterpart $\sim 00^{\prime \prime} 7$ away from the timing position but they conclude that this source is unrelated. Here, we identify a faint source within $0 .{ }^{\prime \prime} 1$ of the pulsar (Gaia 1823773960079216896). We are confident that this is the counterpart to PSR B1957+20 based on available optical photometry (e.g. Reynolds et al. 2007) and the good match between the Gaia and timing proper motion measurements. Combining the negative parallax measurement with DM-based distance estimate, yields $d=1.9_{-0.9}^{+1.0} \mathrm{kpc}$, consistent with the constraints based on spectroscopy (van Kerkwijk et al. 2011).

PSR B1953+29 This system is the second MSP discovered after PSR B 1937+21 and the first one in a binary (Boriakoff et al. 1983). It has a low-mass companion $\left(\lesssim 0.18 \mathrm{M}_{\odot}\right.$; Gonzalez et al. 2011) in a 117 d orbit. Source Gaia 2028584968839606784 was identified as a possible counterpart based on its angular separation of 0. " 1 from the pulsar position, and the low stellar density in the field which implies a small probability for chance alignment $(\leq 3 \%)$. However, the error budget is dominated by the systematic uncertainty which is likely overestimated, given that the timing position is being updated regularly (Arzoumanian et al. 2018, 2020). The same source has been investigated as the possible companion to PSR B1953+29 by Boriakoff et al. (1996) and Mignani et al. (2014). It has a red featureless spectrum suggestive of an evolved star. Indeed, the inferred colors and absolute luminosity place the source above the MS. However, the Gaia parallax measurement implies a distance of $2.3_{-1.0}^{+2.9} \mathrm{kpc}$ which is $\sim 2$ times smaller than the DM-based estimate. In addition, the proper motion does not agree well with that of the pulsar. We therefore conclude that the Gaia source is probably unrelated to the pulsar and that the true companion of the latter is a low-mass WD, as suggested by the radio timing solution.

PSR J1435-6100 This is a $9 \mathrm{~ms}$ pulsar with a massive, $\sim 1 \mathrm{M}_{\odot}$ companion in a $32 \mathrm{~h}$ orbit. This particular combination of fast spin, short orbital period and high companion mass is challenging to explain. The compactness of the orbit suggests that the system is the survivor of a CE episode. To reach the observed spin frequency, the pulsar must have accreted $O\left(0.1 \mathrm{M}_{\odot}\right)$ of material from the donor star (Podsiadlowski et al. 2002; Tauris et al. 2012). This happened either during the $\mathrm{CE}$ event or after, via Case BB mass transfer from the stripped, post-CE companion. In either case, the short evolutionary timescales suggest that the system experienced a phase of supercritical accretion in which a very high accretion flow that led to efficient neutrino cooling (Tauris et al. 2011, 2012).

We identified a $18.9^{m}$ source (Gaia 5878387705005976832 ) 0 .' 3 away from the pulsar timing position. The object has a probability of true association $\sim 95 \%$, and a trigonometric parallax that is consistent with the pulsar DM distance estimate. However, the inferred absolute magnitude of $M_{\mathrm{G}} \simeq-2.7^{\mathrm{m}}$ means that this cannot be the companion responsible for recycling the pulsar. Deep photometric observations obtained by Jacoby et al. (2006) failed to detect any fainter counterpart closer to the pulsar position, which is consistent with the expected WD nature of the companion. Based on that, we conclude that this is either a chance coincidence or, less 
likely, the system is part of a wide triple. The latter possibility can be tested if the pulsar proper motion is measured.

PSRs J1311-3430, J1628-3205 and J2129-0429 Gaia counterparts to these systems were identified by Jennings et al. (2018), but did not show up in our search. The position of PSR J1628-3205 is not well known and hence, it was not included in our final sample. For PSR J1311-3430, the nearest source was filtered out due to its large position error of 2!'9. Similarly, for PSR J2129-0429, the inferred angular separation of the nearest source (1." 3 for Gaia 2672030065446134656 ) was larger than our formal $2 \sigma$ threshold. However, for all these cases the association is confirmed by phaseresolved photometry and spectroscopy. We include these sources in Table 1 for completeness.

PSRs J1843-1113, J1732-5049 and J1949+3106 Possible associations to these pulsars were discussed by Mingarelli et al. (2018). For the nearest source to PSR J1843-1113 (Gaia 4106823440438736384), we infer a separation of $11^{\prime \prime} 44$. Long-term timing of this pulsar (Desvignes et al. 2016) strongly suggests that it is isolated. Hence, we conclude that the Gaia source is most likely unrelated.

For PSRs J1732-5049 and J1949+3106 which are known binaries, we again infer large separations to the proposed counterparts (2!'26 and 2!'82 for Gaia 5946288492263176704 and 2033684267592307584 respectively). In addition, the inferred colors, luminosities and proper motions are inconsistent with the whitedwarf nature of the pulsar companions and the pulsar timing constraints. We therefore conclude that these sources are also unrelated.

\subsection{Young and mildly-recycled pulsars}

Of the 1440 pulsars with $B>10^{9} \mathrm{G}$ in our sample, 11 were found to have a close astrometric pair. Of these, only three are known binaries: PSR J0045-7319 is an LMC pulsar with a B1V companion in a 51 day orbit (Kaspi et al. 1996a,b). PSR B1259-63 is in a highlyeccentric 3.4-yr orbit with a rapidly-rotating OB star (Chernyakova et al. 2014). The system is well known for its 40-day long radio eclipses near periastron. Finally, PSR J2032+4127 is a pulsar in the Cygnus OB2 association. It is in a $\sim 30$-yr orbit around a Be star. It's binary nature was revealed only after long-term timing (Lyne et al. 2015).

In addition to the aforementioned sources, our sample contained a further 59 young and mildly-recycled pulsars in known binaries. Upon investigation we find that all their companions were too faint to be detected by Gaia (van Kerkwijk et al. 2005; Mignani et al. 2014). We identified 8 new close astrometric pairs to which we discuss below.

\section{Candidates}

PSR J0534-6703 This source is a slow pulsar in the LMC (Manchester et al. 2006). We found a bright star within $\sim 0$." 5 of the timing position. Its propoerties are consistent with an early-type MS star at the LMC. The positional offset is similar to the one between PSR J0045-7319 and its optical counterpart. Given that the ATNF timing solution is over 15 years old, this could be attributed to systematics. Interestingly, the pulsar has an unusually high spin period derivative, which might be caused by orbital acceleration. We conclude that a true association between the pulsar and the Gaia source is plausible and cannot be ruled out based on existing information. We therefore recommend further radio timing observations.
PSR J1624-4411 The nearest counterpart to PSR J1624-4411 has a positional offset of 0. " 3 from the timing position (Lorimer et al. 2006). The Gaia source is consistent with an MS star at the inferred distance of $2.7_{-1.8}^{+4.6} \mathrm{kpc}$. The latter also agrees well with the DMbased estimate of $\sim 3.3 \mathrm{kpc}$ and hence a true association is possible.

PSR J1638-4608 Gaia 5992089022760118400 has a separation of only 0 !' 14 from the timing position of this pulsar. Unfortunately, only a 2-parameter astrometric solution is available and hence the true separation might be larger. At the DM-distance of the pulsar, the source would have an absolute magnitude of $M_{\mathrm{G}}=0.3_{-1.6}^{+1.5}$. Interestingly, Kerr et al. (2016) report a 450-day periodic modulation in the timing residuals. If interpreted as a Røemer, the observed amplitude would suggest a planetary companion of a few Earth masses. Such an object would be much fainter than the Gaia source. Under these considerations, we conclude that this is either a chance alignment or that the Gaia source is in a very wide orbit. The next Gaia data releases will hopefully result in more stringent constraints on this system.

PSR J1838-0549 The nearest Gaia entry has an angular separation of $\sim 0$." 5 from the pulsar. Its distance is somewhat smaller but still consistent with the pulsar's DM distance. The inferred distance implies that source is a giant star off the MS. Parthasarathy et al. (2019) recently presented timing data spanning 4 years that show no signs of periodicity. Based on the available information, we conclude that the Gaia source is either in a wide orbit around the pulsar or a foreground star.

PSR J1852+0040 PSR J1852+0040 is a radio-quiet pulsar associated with the SN remnant Kes 79 (Halpern \& Gotthelf 2010). The Gaia source identified in our search has a distance that is only marginally consistent with that of the pulsar. Hence, we conclude that this is either a chance coincidence or that the source is the ejected companion of the pulsar progenitor.

PSR J1903-0258 This pulsar is located 0." 4 away from Gaia 4261581076409458304 (Lorimer et al. 2006). The measured color and luminosity suggest that the latter is an intermediate mass MS star. Given that the timing solution has not been updated for over 15 years, the two objects may be physically associated.

PSR J1958+2846 This is a radio-quiet pulsar that has been timed using the Fermi-LAT instrument. The $\gamma$-ray timing solution (Kerr et al. 2016) derived using $~ 1.5$-yr of data shows no sign of binarity. However, given the low accuracy of the time-of-arrival measurements and the small span of the data, an association with Gaia 2030000280820200960 , located 0." 4 from the formal position is still possible.

PSR J2027+4557 This pulsar was found in a radio survey of Cygnus OB associations (Janssen et al. 2009). The Gaia source that showed up in our search is consistent with an early-type MS star at the inferred distance of $1.96_{-0.20}^{+0.25} \mathrm{kpc}$. The latter is smaller than the DM distance estimate. The pulsar bears similarities to PSR J2032+4127, located inside the Cygnus OB2 region. The latter has a DM of $115 \mathrm{pc} \mathrm{cm}^{-3}$, i.e. $\sim 2$ times smaller. Hence, a local un-modelled contribution to the DM would be needed, which is not impossible given the complexity of the region. 


\section{THE MULTIPLICITY FRACTION AMONG YOUNG PULSARS}

The results presented in Section 3 confirm that the observed multiplicity fraction (henceforth $f_{\text {young }}^{\text {obs }}$ ) among young pulsars is small: of the 1377 galactic pulsars ${ }^{4}$ with $B>10^{10} \mathrm{G}$ in our sample, only 22(16) are in binaries with(without) considering the candidates described in the previous section, thus $f_{\text {young }}^{\text {obs }}=1.16-1.59 \%$. The latter is considerably smaller than the multiplicity fraction among MSPs and mild-MSPs ( $f_{\text {MSP }}^{\text {obs }} \simeq 79 \%$ and $f_{\text {mild }}^{\text {obs }} \simeq 85 \%$ respectively).

In interpreting these results, one has to consider two strong selection effects: 1) Radio timing is not sensitive to binaries with orbital periods considerably longer than the observational timespan and Røemer delays much smaller than the amplitude of the timing noise, and b) Gaia cannot detect sources fainter than $m_{\mathrm{g}} \gtrsim 20.5^{\mathrm{m}}$. To examine the impact of these biases and place a constraint on the true multiplicity fraction among young pulsars, $f_{\text {young }}^{\text {true }}$, we constructed a toy model to simulate various scenarios for the orbital and optical properties of possible companions stars and estimate their detectability at radio and optical wavelengths.

In these simulations, we split the 1377 young Galactic pulsars in our sample in three categories: the first contained all known binaries $\left(P_{\mathrm{b}} \geq 0\right.$ in PSRCat), the second the seven Galactic candidates of Table 1 and the third, the remaining seemingly isolated pulsars. We then used a Monte-Carlo sampling scheme with $10^{5}$ iterations to estimate the number of binaries in each group and infer the probability distribution for $f_{\text {young }}^{\text {true }}$. All pulsars belonging to the first group were obviously always counted as binaries. For the second group, we decided on whether the candidates of Table 1 are true binary companions using their inferred probabilities of chance coincidence. For the third group, we assigned a companion with a separation and mass drawn from various astrophysically-motivated distributions (see below). The brightness was inferred using a massluminosity relation for MS stars, the extinction curves of Green et al. (2019) (see Section 3), and a distance normally distributed about the DM distance with a fractional uncertainty of $20 \%$. The multiplicity fraction for each sample population was inferred by counting all binaries in the first two categories and adding those systems from the final category that had orbital periods and apparent magnitudes above a certain detectability threshold, $P_{\mathrm{b}} \geq P_{\mathrm{b}}^{\text {thres }}$ and $m_{\mathrm{g}} \geq m_{\mathrm{g}}^{\text {thres }}$.

Figure 3 shows the results for various simulated scenarios. For our fiducial model (shown in blue), companion masses were inferred by combining a Salpeter mass function in the $10 . \ldots 50 \mathrm{M}_{\odot}$ range for the pulsar progenitors, with the best-fit mass ratio distribution of Sana et al. (2012). Orbital periods ranging from $1 \mathrm{~d}$ to $1000 \mathrm{yr}$, were also distributed according to the results of the latter work, while for the Gaia and radio timing detectability thresholds we assumed $P_{\mathrm{b}}^{\text {thres }}=50 \mathrm{yr}$ and $m_{\mathrm{g}}^{\text {thres }}=20.5^{\mathrm{m}}$. This scenario - which we consider to be the most realistic, given all underlying uncertainties places an upper limit of $b_{\text {young }}^{\text {true }} \leq 5.3 \%$ at the $99.8 \%$ confidence level (CL). Radio timing appears to be the most sensitive probe of binarity $\left(f_{\text {young }}^{\text {true }} \leq 8.3 \%\right.$ at $99.8 \% \mathrm{CL}$ for $P_{\mathrm{b}}^{\text {thres }}=10 \mathrm{yr}$ ), while massive companions would be the easiest to detect with Gaia $\left(f_{\text {young }}^{\mathrm{OB}} \leq 3.6 \%\right.$ at $99.8 \% \mathrm{CL}$ for early-type companions brighter than $\left.M_{g}=-2.6^{\mathrm{M}}\right)$. Overall, we find that the binary fraction could still be $\sim 3$ to 8 times higher than the observed one, given present limitations.

An additional selection effect against pulsars in binaries that is unaccounted for in the aforementioned models is the stellar wind of

\footnotetext{
4 (here we do not consider extragalactic pulsars, since some of the processes impacting $f_{\text {young }}^{\text {true }}$ depend strongly on metallicity)
}

the companion which may be opaque to radio emission. The high mass-loss rates expected for upper-MS stars could create a dense environment leading to free-free absorption. For pulsars in our sample, this means that certain combinations of orbits and companion masses are ruled out (given that a pulsar is observed). For a circular orbit (i.e. constant separation), the minimum period below which $21-\mathrm{cm}$ radiation would be eclipsed by a $1000 \mathrm{~km} \mathrm{~s}^{-1}$ outflow with a temperature of $10^{4} \mathrm{~K}$ (Illarionov \& Sunyaev 1975) is:

$$
P_{\mathrm{b}}^{\min } \simeq 0.28\left(\frac{\dot{M}}{4.8 \times 10^{-12} \mathrm{M}_{\odot} \mathrm{yr}^{-1}}\right)\left(\frac{M_{\mathrm{tot}}}{\mathrm{M}_{\odot}}\right)^{-1} \text { days }
$$

where $\dot{M}$ is the wind mass-loss rate and $\mathrm{M}_{\text {tot }}$ is the total mass of the binary. In practice, we find that taking this effect into account in our toy model - assuming standard mass-loss recipes for the MS (Kippenhahn et al. 2012) — has a very small impact on the results described above (see dashed lines in Figure 3). This is because freefree absorption only becomes relevant for large companion masses and short separations. For the models discussed here, most such systems are assumed to be within the sensitivity of exiting timing programs and are thus already ruled out. However, Eq. 4 also suggests that some rotation-powered pulsars with massive companions are obscured at radio wavelengths and therefore, the intrinsic binary fraction could be even larger. Some clues about the influence of this effect is obtained from the properties of known systems: Of the pulsars investigated here, only the 1237-day binary PSR B1259-63 is eclipsed over $\sim 3 \%$ of its orbit near periastron (Johnston et al. 1992, 1994, 2005). Considering the shape of the mass function, this suggests that stellar winds should only affect a small fraction of pulsars when they approach their massive companions (either due to compact orbits or high eccentricities). On the other hand, pulsars in sufficiently close orbits around massive stars would become wind-fed high-mass X-ray binaries (HMXBs). Indeed, most known wind-fed HMXBs have orbital periods in the 3...300 d range and $\mathrm{X}$-ray luminosities that roughly scale as $L_{\mathrm{x}} \propto P_{\mathrm{b}}^{-4 / 3}$, as expected for spherical accretion (e.g. Lutovinov et al. 2013; Chaty 2013; Walter et al. 2015; Tauris et al. 2017, and references therein). However, these sources are accretion-powered and hence not directly relevant to the population of pulsars considered here.

To conclude, we believe that absorption is only relevant to a small fraction of pulsars orbiting massive companions with periods of up to few years. To be hidden, these pulsars must approach their companions sufficiently close to intersect the optically-thick part of the wind, but not too close so as to become persistent HMXBs. In turn, this means that the constraints on $f_{\text {young }}^{\text {true }}$ inferred in this section should be a reliable proxy of the true frequency of pulsars in stellar binaries.

\section{CONCLUSIONS AND PROSPECTS}

We have performed a search for optical counterparts to 1534 rotation-powered pulsars. By utilizing the Gaia DR2 catalogue and back-propagating its astrometric solution to the reference epoch of each pulsar's ephemeris (Section 2), we were able to search for close astrometric pairs. We identified 20 previously known binary companions - the majority of which are companions to MSPs — and 8 tentative new matches to young pulsars. For these candidates, we find that their Gaia parallaxes are broadly consistent with the pulsar distance estimates and recommend further timing observations to test if they are true binary companions.

Most MSP companions in Gaia DR2 are early-type sub-dwarfs 


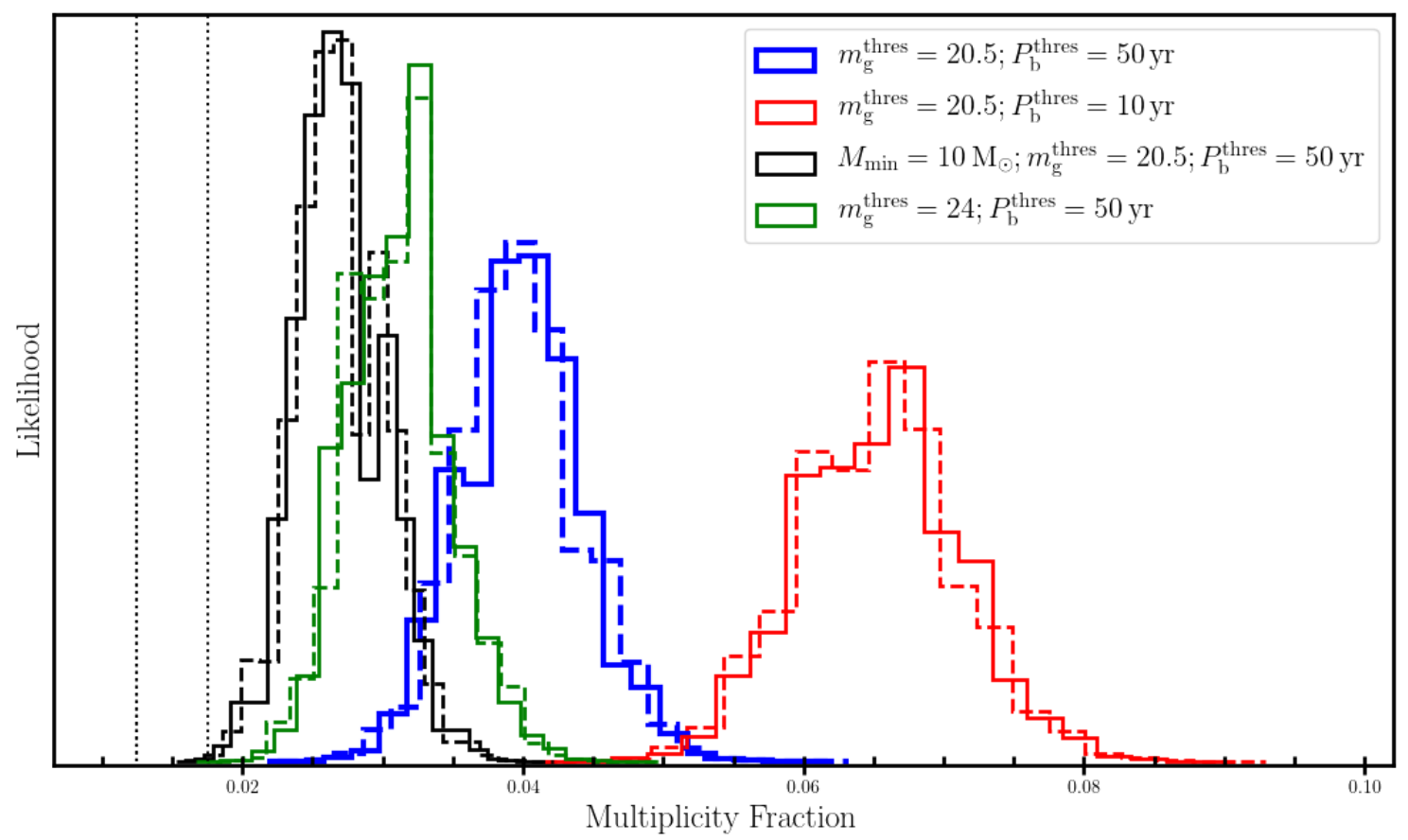

Figure 3. The probability density function for $f_{\text {young }}^{\text {true }}$ given different detectability thresholds and scenarios discussed in the text (see Sections 4 and 5). Dashed lines show the results of simulations in which the possibility of free-free absorbtion of pulsar emission from the stellar wind is taken into account.

significantly bluer and more luminous than MS stars with similar masses. Their colours, luminosities and reduced proper motions sets them apart from the majority of stars on the HR and RPM diagrams. In Section 3.1, we inferred empirical color-magnitude- $\mathrm{H}_{\mathrm{g}}$ criteria (Equation 3) that satisfy the observed properties of eclipsing and WD companions to MSPs and filter out $\gtrsim 99.8 \%$ of field stars. While some contamination is expected by sdA stars (Pelisoli et al. 2019b; Pelisoli \& Vos 2019; Pelisoli et al. 2019a), these selection criteria allow to single out Gaia sources that might be orbited by neutron stars. Deep targeted radio surveys of these candidates might be proven a viable path towards discovering new MSPs. Because of the information provided by Gaia, pulsars discovered in this way would be extremely valuable for a broad range of astrophysical inquiry (Antoniadis et al. 2012; Mingarelli et al. 2018; Arzoumanian et al. 2020). For instance, MSPs with bright WD companions enable precise NS mass measurements, which are important for constraining the mass function of compact stars and the equation-of-state of super-dense matter (Antoniadis et al. 2013; Antoniadis et al. 2016). Similarly, eclipsing MSPs are key in understanding NS recycling and the end-states of low-mass XRBs (e.g. van Kerkwijk et al. 2011; Tauris et al. 2015a; Strader et al. 2019, and references therein).

In Section 4, we used a toy model to investigate the impact of observational selection effects on the detectability of pulsar companions. We constrained the true multiplicity fraction among young pulsars to be $\leq 5.3 \%$ under the assumption that all binaries with orbital periods shorter than 50 years and companions brighter than $20.5^{m}$ would have been detected by Gaia and ongoing timing programs. We also concluded that the number of pulsars with longer orbital periods that are obscured by the wind of their companions should be small.

Given that our toy model only considers stellar binaries, the number of pulsars with planetary companions could still be considerably larger. Indeed, low-mass objects are considered as the possible cause of the low-amplitude periodic signals found in the timing residuals of several young pulsars. (e.g. Ray et al. 2011; Parthasarathy et al. 2019). With this caveat in mind, our constraints on $f_{\text {young }}^{\text {true }}$ should be reliable proxy for the number of young pulsars orbited by stars. While our analysis confirms that $f_{\text {young }}^{\text {true }}$ is low, we find that it could still be a factor 5-8 larger than previously thought. Hence, there could still be of order $\sim 100$ hidden binaries in the known pulsar population. For massive companions in particular, we inferred a more stringent limit of $f_{\mathrm{OB}}^{\text {true }} \leq 3.7 \%$. Combined with the Milky-Way core-collapse SN rate $\left(r_{\mathrm{SN}}=0.01-0.02 \mathrm{yr}^{-1}\right.$, see Diehl et al. 2006; Keane \& Kramer 2008, and references therein), this places a very conservative, but independent upper limit on the Galactic NS coalescence rate, $r_{\text {mergers }} \lesssim f_{\mathrm{OB}}^{\text {true }} \times r_{\mathrm{SN}}=(3.7-7.4) \times 10^{-4} \mathrm{yr}^{-1}$ (with the caveat that some close binaries may be HMXBs, see Section 4). The former is consistent with (but more conservative than) the more direct constraints based on double neutron star systems (Kalogera et al. 2004; Pol et al. 2020), the observed number of HMXBs in the Galaxy (Tauris et al. 2017) and the advanced LIGO/Virgo constraint of $\left(3.2_{-2.4}^{+4.9}\right) \times 10^{-5} \mathrm{yr}^{-1}$ (Abbott et al. 2020).

There are several evolutionary pathways leading to the formation of isolated young pulsars. Theoretical models predict that while $\sim$ one third originate from single stars or binaries that merge during the MS (Portegies Zwart \& Yungelson 1999), the majority 
must be due to $\mathrm{SNe}$ in massive binary systems. The relative number of pulsars released during the first and second explosion, as well as the frequency of surviving binaries depends sensitively on the magnitude distribution of natal SN kicks. A binary system with a given separation is more likely to survive a kick of a certain magnitude during the first $\mathrm{SN}$ explosion, when the secondary is still a massive star. The second SN can then release up to two NSs (one young and one midly recycled). In this case one would expect a relatively large number of isolated mild MSPs, which appears to disagree with observations ( $f_{\text {mild }}^{\text {obs }} \simeq 85 \%$, see Section 4 ). This suggests that most young pulsars originate from the first $\mathrm{SN}$ in a wide binary, or the released secondary star. On the other hand, compact orbits, stripping of the secondary star prior to its core collapse and electron-capture SNe lead to increased binary survival rates and therefore, they can help explain the properties of the observed binary pulsar population (Podsiadlowski et al. 2004; Tauris et al. 2013, 2015 b, 2017). Nevertheless our results appear to be in broad agreement with kick amplitudes of few $100 \mathrm{~km} \mathrm{~s}^{-1}$ for core-collapse SNe (Schneider et al. 2020), while extremely small-amplitude kicks are disfavoured given the small number of pulsars in wide binaries (see also Igoshev \& Perets 2019). Detailed population-synthesis studies and comparison with all available observational benchmarks will be required to reach more quantitative conclusions.

It is evident that this study will benefit significantly by future iterations of the Gaia catalogue that will provide improved astrometry for a larger number of stars. Next-generation surveys such as the Vera C. Rubin Large Synoptic Survey Telescope (Kessler et al. 2019) will be even more important in identifying dimmer and less massive pulsar companions (Figure 3 ) as well as optical emission from young pulsars similar to the Crab. Close astrometric pairs - even those that are not true binary companions - can also be promising targets for pulsar scintillation studies (Simard \& Pen 2018; Main et al. 2018, 2020). Finally, an extension of the method presented here combined with improved astrometry will also allow to identify potential future micro-lensing events (Dai et al. 2015) and stars ejected during SNe.

Despite the current importance of optical astrometry in constraining the frequency of binary pulsars, our simulation suggest that pulsar timing remains the most sensitive probe of multiplicity. Until recently, regular monitoring of all known pulsars was impractical due to limited telescope availability and resources. The advent of large-scale monitoring projects such as the CHIME/pulsar project (Ng 2018; Ng et al. 2020) and its southern counterpart HIRAX (Newburgh et al. 2016), MeerTime (Bailes et al. 2016, 2020; Johnston et al. 2020) and eventually the Square Kilometre Array (Tauris et al. 2015a; Antoniadis et al. 2015) will therefore lead to significantly more stringent constraints on the young pulsar multiplicity fraction in the near future.

\section{DATA AVAILABILITY}

The python/jupyter notebook to reproduce the results of this article is available at https://dx.doi.org/10.5281/zenodo. 4075042 .

\section{ACKNOWLEDGEMENTS}

Support was provided by the Hellenic Foundation for Research and Innovation (HFRI) and the Stavros Niarchos Foundation (SNF) under grant agreement No.72-1/11.8.2020. I am grateful to the referee for their constructive report. I am also grateful to Philipp Podsiadlowski, Paulo Freire, Michael Kramer and Vivek Venkatraman Krishnan for discussions. This work relies on data from the European Space Agency (ESA) mission Gaia (https://www . cosmos.esa.int/gaia), processed by the Gaia Data Processing and Analysis Consortium (DPAC, https://www.cosmos.esa. int/web/gaia/dpac/consortium). This research made use of NumPy (Harris et al. 2020), Matplotlib (Hunter 2007) and Astropy (http://www . astropy . org), a community-developed core Python package for Astronomy (Astropy Collaboration et al. 2013, 2018) and of NASA's Astrophysics Data System Bibliographic Services. 


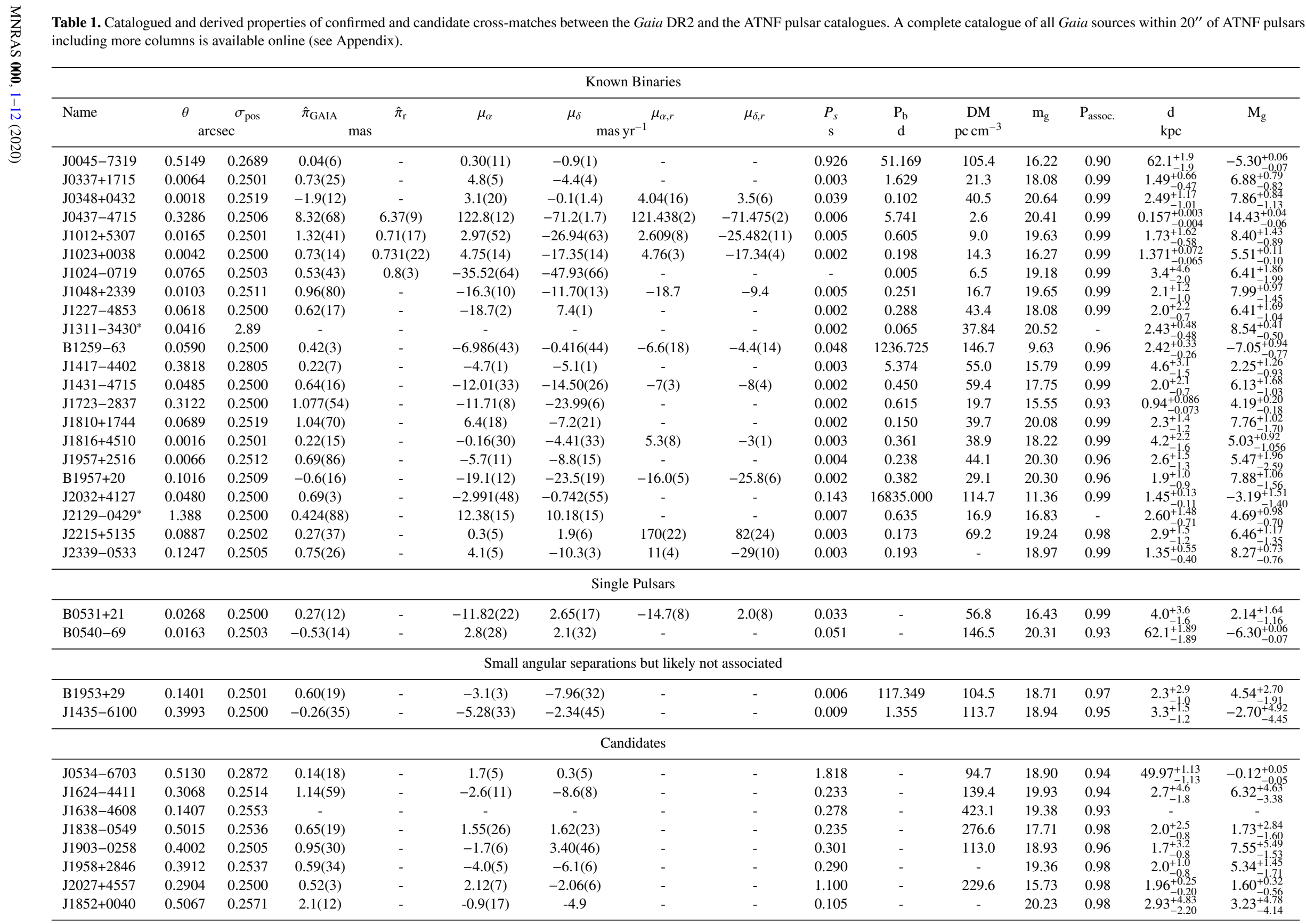




\section{APPENDIX A: DESCRIPTION OF COLUMNS IN ONLINE CATALOGUE}

The full catalogue of sources withing $20^{\prime \prime}$ of ATNF pulsars is available on VizieR (http://cdsarc.u-strasbg.fr/viz-bin/). The table below provides a summary of the information available in the catalogue.

\section{REFERENCES}

Abadie J., et al., 2010, Classical and Quantum Gravity, 27, 173001

Abbott B. P., et al., 2017, Physical Review Letters, 119, 161101

Abbott R., et al., 2020, arXiv e-prints, 2010, arXiv:2010.14533

Antoniadis J., Bassa C. G., Wex N., Kramer M., Napiwotzki R., 2011, Monthly Notices of the Royal Astronomical Society, 412, 580

Antoniadis J., van Kerkwijk M. H., Koester D., Freire P. C. C., Wex N., Tauris T. M., Kramer M., Bassa C. G., 2012, Monthly Notices of the Royal Astronomical Society, 423, 3316

Antoniadis J., et al., 2013, Science, 340, 448

Antoniadis J., et al., 2015, Advancing Astrophysics with the Square Kilometre Array (AASKA14), p. 157

Antoniadis J., Tauris T. M., Ozel F., Barr E., Champion D. J., Freire P. C. C., 2016, arXiv e-prints, 1605, arXiv:1605.01665

Arzoumanian Z., et al., 2018, The Astrophysical Journal Supplement Series, 235,37

Arzoumanian Z., et al., 2020, arXiv e-prints, 2009, arXiv:2009.04496

Astraatmadja T. L., Bailer-Jones C. A. L., 2016, The Astrophysical Journal, 832, 137

Astropy Collaboration et al., 2013, Astronomy and Astrophysics, 558, A33

Astropy Collaboration et al., 2018, The Astronomical Journal, 156, 123

Bailer-Jones C. A. L., 2015, PASP, 127, 994

Bailer-Jones C. A. L., Rybizki J., Fouesneau M., Mantelet G., Andrae R., 2018, The Astronomical Journal, 156, 58

Bailes M., 1989, The Astrophysical Journal, 342, 917

Bailes M., et al., 2016, arXiv:1803.07424, p. 11

Bailes M., et al., 2020, Publications of the Astronomical Society of Australia, $37, \mathrm{e} 028$

Bassa C. G., et al., 2016, Monthly Notices of the Royal Astronomical Society, 460, 2207

Boriakoff V., Buccheri R., Fauci F., 1983, Nature, 304, 417

Boriakoff V., Beskin G., Dossa D., Fauci F., Oke J. B., Tella L., 1996, in Johnston S., Walker M. A., Bailes M., eds, Astronomical Society of the Pacific Conference Series Vol. 105, IAU Colloq. 160: Pulsars: Problems and Progress. p. 303

Chaty S., 2013, Advances in Space Research, 52, 2132

Chernyakova M., et al., 2014, Monthly Notices of the Royal Astronomical Society, 439, 432

Dai S., Smith M. C., Lin M. X., Yue Y. L., Hobbs G., Xu R. X., 2015, The Astrophysical Journal, 802, 120

Deller A. T., et al., 2019, The Astrophysical Journal, 875, 100

Desvignes G., et al., 2016, Monthly Notices of the Royal Astronomical Society, 458,3341

Diehl R., et al., 2006, Nature, 439, 45

Ding H., Deller A. T., Freire P., Kaplan D. L., Lazio T. J. W., Shannon R., Stappers B., 2020, The Astrophysical Journal, 896, 85

Ferdman R. D., et al., 2020, Nature, 583, 211

Gaia Collaboration et al., 2016, Astronomy and Astrophysics, 595, A1

Gonzalez M. E., et al., 2011, The Astrophysical Journal, 743, 102

Graczyk D., et al., 2013, The Astrophysical Journal, 780, 59

Green G. M., 2018, Journal of Open Source Software, 3, 695

Green G. M., et al., 2014, The Astrophysical Journal, 783, 114

Green G. M., Schlafly E., Zucker C., Speagle J. S., Finkbeiner D., 2019, The Astrophysical Journal, 887, 93

Halpern J. P., Gotthelf E. V., 2010, The Astrophysical Journal, 709, 436

Harris C. R., et al., 2020, Nature, 585, 357-362

Hobbs G., Lorimer D. R., Lyne A. G., Kramer M., 2005, Monthly Notices of the Royal Astronomical Society, 360, 974
Hunter J. D., 2007, Computing in Science Engineering, 9, 90

Igoshev A. P., Perets H. B., 2019, Monthly Notices of the Royal Astronomical Society, 486, 4098

Illarionov A. F., Sunyaev R. A., 1975, Astronomy and Astrophysics, 39, 185 Ivanova N., et al., 2013, Astronomy and Astrophysics Review, 21, 59

Jacoby B. A., Chakrabarty D., van Kerkwijk M. H., Kulkarni S. R., Kaplan D. L., 2006, The Astrophysical Journal Letters, 640, L183

Janka H.-T., 2013, Monthly Notices of the Royal Astronomical Society, 434, 1355

Janssen G. H., Stappers B. W., Braun R., van Straten W., Edwards R. T., Rubio-Herrera E., van Leeuwen J., Weltevrede P., 2009, Astronomy and Astrophysics, 498, 223

Jennings R. J., Kaplan D. L., Chatterjee S., Cordes J. M., Deller A. T., 2018, The Astrophysical Journal, 864, 26

Johnston S., Manchester R. N., Lyne A. G., Bailes M., Kaspi V. M., Qiao G., D'Amico N., 1992, The Astrophysical Journal Letters, 387, L37

Johnston S., Manchester R. N., Lyne A. G., Nicastro L., Spyromilio J., 1994, Monthly Notices of the Royal Astronomical Society, 268, 430

Johnston S., Ball L., Wang N., Manchester R. N., 2005, Monthly Notices of the Royal Astronomical Society, 358, 1069

Johnston S., et al., 2020, Monthly Notices of the Royal Astronomical Society, 493, 3608

Kalogera V., et al., 2004, The Astrophysical Journal Letters, 601, L179

Kaplan D. L., et al., 2016, The Astrophysical Journal, 826, 86

Kaspi V. M., Bailes M., Manchester R. N., Stappers B. W., Bell J. F., 1996a, Nature, 381, 584

Kaspi V. M., Tauris T. M., Manchester R. N., 1996b, The Astrophysical Journal, 459, 717

Keane E. F., Kramer M., 2008, Monthly Notices of the Royal Astronomical Society, 391, 2009

Kerr M., Hobbs G., Johnston S., Shannon R. M., 2016, Monthly Notices of the Royal Astronomical Society, 455, 1845

Kessler R., et al., 2019, arXiv:1903.11756 [astro-ph]

Kippenhahn R., Weigert A., Weiss A., 2012, Stellar Structure and Evolution, second edn. Astronomy and Astrophysics Library, Springer-Verlag, Berlin Heidelberg, doi:10.1007/978-3-642-30304-3

Lindegren L., et al., 2018, Astronomy \& Astrophysics, 616, A2

Lorimer D. R., et al., 2006, Monthly Notices of the Royal Astronomical Society, 372, 777

Luri X., et al., 2018, Astronomy \& Astrophysics, 616, A9

Lutovinov A. A., Revnivtsev M. G., Tsygankov S. S., Krivonos R. A., 2013, Monthly Notices of the Royal Astronomical Society, 431, 327

Lutz T. E., Kelker D. H., 1973, PASP, 85, 573

Luyten W. J., 1922, Lick Observatory Bulletin, 10, 135

Lyne A. G., Stappers B. W., Keith M. J., Ray P. S., Kerr M., Camilo F., Johnson T. J., 2015, Monthly Notices of the Royal Astronomical Society, 451,581

Main R., et al., 2018, Nature, 557, 522

Main R. A., et al., 2020, arXiv e-prints, 2009, arXiv:2009.10707

Manchester R. N., Hobbs G. B., Teoh A., Hobbs M., 2005, The Astronomical Journal, 129, 1993

Manchester R. N., Fan G., Lyne A. G., Kaspi V. M., Crawford F., 2006, The Astrophysical Journal, 649, 235

Mignani R. P., et al., 2014, Monthly Notices of the Royal Astronomical Society, 443, 2223

Mignard F., et al., 2018, Astronomy \& Astrophysics, 616, A14

Mingarelli C. M. F., Anderson L., Bedell M., Spergel D. N., 2018, arXiv e-prints, 1812, arXiv:1812.06262

Newburgh L. B., et al., 2016, arXiv:1607.02059, 9906, 99065X

Ng C., 2018, arXiv:1711.02104, 337, 179

Ng C., et al., 2020, Monthly Notices of the Royal Astronomical Society, 496, 2836

Parthasarathy A., et al., 2019, Monthly Notices of the Royal Astronomical Society, 489, 3810

Pelisoli I., Vos J., 2019, Monthly Notices of the Royal Astronomical Society, 488,2892

Pelisoli I., Bell K. J., Kepler S. O., Koester D., 2019a, Monthly Notices of the Royal Astronomical Society, 482, 3831 
Table A1. Catalogue Format

\begin{tabular}{|c|c|c|}
\hline Name & Format & Description \\
\hline angDist & float64 & angular Separation from the pulsar (in degrees) \\
\hline id & int64 & pulsar identification number \\
\hline name & $<\mathrm{U} 12$ & pulsar name \\
\hline survey & $<\mathrm{U} 48$ & survey that discovered the pulsar as defined in psrcat \\
\hline BinComp & $<\mathrm{U} 6$ & companion type as defined in psrcat \\
\hline Type & int64 & pulsar type as defined in psrcat \\
\hline $\mathrm{raj}$ & float64 & pulsar right ascension at PosEpoch (in degrees) \\
\hline rajerr & float64 & error in pulsar right ascension at PosEpoch (in degrees) \\
\hline $\operatorname{dec} j$ & float64 & pulsar right ascension at PosEpoch (in degrees) \\
\hline decjerr & float64 & error in pulsar right ascension at PosEpoch (in degrees) \\
\hline pmra_radio & float64 & radio timing proper motion in $\mathrm{RA}(\mathrm{mas} / \mathrm{yr})$ \\
\hline pmraerr & float64 & error of radio timing proper motion in RA (mas/yr) \\
\hline pmdec_radio & float64 & radio timing proper motion in DEC (mas/yr) \\
\hline pmdecerr & float64 & error of radio timing proper motion in DEC (mas/yr) \\
\hline $\mathrm{px}$ & float64 & radio timing parallax (mas) \\
\hline pxerr & float64 & error of radio timing parallax (mas) \\
\hline posepoch & float64 & position epoch of the radio timing solution \\
\hline po & float64 & spin period $(\mathrm{s})$ \\
\hline pQerr & float64 & error of spin period (s) \\
\hline p1 & float64 & spin-period derivative \\
\hline p1err & float64 & error of spin-period derivative \\
\hline $\mathrm{dm}$ & float64 & dispersion measure $\left(\mathrm{pc} / \mathrm{cm}^{3}\right)$ \\
\hline dmerr & float64 & error of dispersion measure $\left(\mathrm{pc} / \mathrm{cm}^{3}\right)$ \\
\hline dist & float64 & DM distance estimate (see text) \\
\hline $\mathrm{pb}$ & float64 & orbital period (days) \\
\hline pberr & float64 & error of orbital period (days) \\
\hline raj_now & float64 & RA of source at posepoch \\
\hline decj_now & float64 & DEC of source at posepoch \\
\hline ra_epoch 2000 & float64 & RA of source at $\mathrm{J} 2000$ \\
\hline dec_epoch2000 & float64 & DEC of source at $\mathrm{J} 2000$ \\
\hline source_id & int64 & GAIA source ID \\
\hline $\mathrm{ra}$ & float64 & RA of source at $\mathrm{J} 2015.5$ \\
\hline ra_error & float64 & RA uncertainty of source at $\mathrm{J} 2000$ \\
\hline dec & float64 & DEC of source at $\mathrm{J} 2000$ \\
\hline dec_error & float64 & DEC uncertainty of source at $\mathrm{J} 2000$ \\
\hline parallax & float64 & Gaia parallax (mas) \\
\hline parallax_error & float64 & Gaia parallax error (mas) \\
\hline pmra & float64 & Gaia proper motion in RA (mas/yr) \\
\hline pmra_error & float64 & error of Gaia proper motion in RA (mas/yr) \\
\hline pmdec & float64 & Gaia proper motion in DEC (mas/yr) \\
\hline pmdec_error & float64 & error of Gaia proper motion in DEC (mas/yr) \\
\hline phot_g_mean_mag & float64 & Gaia mean magnitude \\
\hline phot_bp_mean_mag & float64 & Gaia mean magnitude in BP band \\
\hline phot_rp_mean_mag & float64 & Gaia mean magnitude in RP band \\
\hline bp_rp & float64 & Gaia BP-RP mean color \\
\hline poserror & float64 & position error of the source at posepoch $(\operatorname{arcsec})$ \\
\hline poserror_sys & float64 & position error of the source including systematics at posepoch (arcsec) \\
\hline angDist & float64 & angular separation from the nearest pulsar $(\operatorname{arcsec})$ \\
\hline TrueAngDist & float64 & angular separation from the nearest pulsar at posepoch (arcsec) \\
\hline Pass & float64 & probability of Association (1 - Probability of chance coincidence) \\
\hline g_abs* & float64 & Absolute $\mathrm{G}$ magnitude corrected for extinction \\
\hline b_r_abs* & float64 & $B-R$ color corrected for extinction \\
\hline distance* & float64 & Median distance $(\mathrm{kpc})$ \\
\hline distance_min* & float64 & $16 \%$ lower bound on distance $(\mathrm{kpc})$ \\
\hline distance_max* & float64 & $68 \%$ upper bound on distance $(\mathrm{kpc})$ \\
\hline dust & float64 & median $\mathrm{E}(\mathrm{B}-\mathrm{V})$ extinction \\
\hline dust_min & float64 & $16 \%$ lower bound on extinction \\
\hline dust_max & float64 & $68 \%$ upper bound on extinction \\
\hline
\end{tabular}

* Calculated only for the sources in Table 1 


\section{J. Antoniadis}

Pelisoli I., Bell K. J., Kepler S. O., Koester D., 2019b, Monthly Notices of the Royal Astronomical Society, 482, 3831

Pietrzyński G., et al., 2013, Nature, 495, 76

Podsiadlowski P., Rappaport S., Pfahl E. D., 2002, The Astrophysical Journal, 565, 1107

Podsiadlowski P., Langer N., Poelarends A. J. T., Rappaport S., Heger A., Pfahl E., 2004, The Astrophysical Journal, 612, 1044

Pol N., McLaughlin M., Lorimer D. R., 2020, Research Notes of the AAS, 4, 22

Portegies Zwart S. F., Yungelson L. R., 1999, Monthly Notices of the Royal Astronomical Society, 309, 26

Ray P. S., et al., 2011, The Astrophysical Journal Supplement Series, 194, 17

Reynolds M. T., Callanan P. J., Fruchter A. S., Torres M. a. P., Beer M. E., Gibbons R. A., 2007, Monthly Notices of the Royal Astronomical Society, 379, 1117

Sana H., et al., 2012, Science, 337, 444

Schneider F. R. N., Podsiadlowski P., Müller B., 2020, arXiv e-prints, 2008, arXiv:2008.08599

Simard D., Pen U.-L., 2018, Monthly Notices of the Royal Astronomical Society, 478, 983

Strader J., et al., 2019, The Astrophysical Journal, 872, 42

Tauris T. M., Langer N., Kramer M., 2011, Monthly Notices of the Royal Astronomical Society, 416, 2130

Tauris T. M., Langer N., Kramer M., 2012, Monthly Notices of the Royal Astronomical Society, Volume 425, Issue 3, pp. 1601-1627., 425, 1601

Tauris T. M., Langer N., Moriya T. J., Podsiadlowski P., Yoon S.-C., Blinnikov S. I., 2013, The Astrophysical Journal Letters, 778, L23

Tauris T. M., et al., 2015a, Advancing Astrophysics with the Square Kilometre Array (AASKA14), p. 39

Tauris T. M., Langer N., Podsiadlowski P., 2015b, Monthly Notices of the Royal Astronomical Society, 451, 2123

Tauris T. M., et al., 2017, ApJ, 846, 170

Walter R., Lutovinov A. A., Bozzo E., Tsygankov S. S., 2015, Astronomy and Astrophysics Review, 23, 2

van Kerkwijk M. H., Bassa C. G., Jacoby B. A., Jonker P. G., 2005, in Rasio F. A., Stairs I. H., eds, Astronomical Society of the Pacific Conference Series Vol. 328, Binary Radio Pulsars. p. 357 (arXiv:astro-ph/0405283)

van Kerkwijk M. H., Breton R. P., Kulkarni S. R., 2011, The Astrophysical Journal, 728, 95

This paper has been typeset from a $\mathrm{T}_{\mathrm{E}} \mathrm{X} / \mathrm{L} \mathrm{AT} \mathrm{E}$ file prepared by the author. 